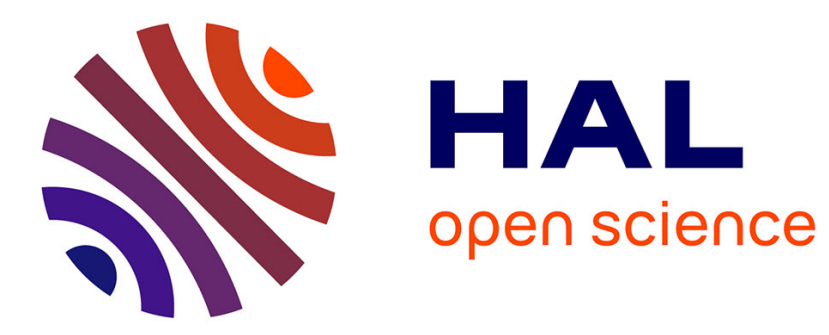

\title{
Continuous-discrete adaptive observers for state affine systems
}

Tarek Ahmed-Ali, Romain Postoyan, Françoise Lamnabhi-Lagarrigue

\section{To cite this version:}

Tarek Ahmed-Ali, Romain Postoyan, Françoise Lamnabhi-Lagarrigue. Continuous-discrete adaptive observers for state affine systems. Automatica, 2009, 45 (12), pp.2986-2990. 10.1016/j.automatica.2009.09.005 . hal-01058652

\section{HAL Id: hal-01058652 \\ https://hal.science/hal-01058652}

Submitted on 27 Aug 2014

HAL is a multi-disciplinary open access archive for the deposit and dissemination of scientific research documents, whether they are published or not. The documents may come from teaching and research institutions in France or abroad, or from public or private research centers.
L'archive ouverte pluridisciplinaire HAL, est destinée au dépôt et à la diffusion de documents scientifiques de niveau recherche, publiés ou non, émanant des établissements d'enseignement et de recherche français ou étrangers, des laboratoires publics ou privés. 


\title{
Continuous-discrete adaptive observers for state affine systems
}

\author{
Tarek Ahmed-Ali ${ }^{\mathrm{a}, *}$, Romain Postoyan ${ }^{\mathrm{b}}$, Françoise Lamnabhi-Lagarrigue ${ }^{\mathrm{c}}$ \\ a Univ Caen, GREYC-ENSICAEN, UMR CNRS 6072- 6 Boulevard du Maréchal Juin, 14050 Caen Cedex, France \\ b Univ Paris-Sud, LSS-CNRS Supélec, 3 rue Joliot Curie, 91192 Gif-sur-Yvette, France \\ ' EECI, LSS-CNRS Supélec, 3 rue Joliot Curie, 91192 Gif-sur-Yvette, France
}

\begin{abstract}
A B S T R A C T
The observation of a class of multi-input multi-output (MIMO) state affine systems with constant unknown parameters and discrete time output measurements is addressed. Assuming some persistent excitation conditions to hold and the sampling steps to satisfy some boundedness hypotheses, system observability is ensured and a class of global exponential observers is synthesized.
\end{abstract}

Keywords:

Sampled-data observer

Adaptive observer

\section{Introduction}

The synthesis of observers for continuous-time systems with sampled measurements has received much attention over the past decades. A classical solution used to handle the discrete nature of the system output consists either in appropriately discretizing a known continuous time observer (Arcak and Nešić, 2004) or in synthesizing a discrete-time observer for a consistent approximation of the exact discretized system (Arcak and Nešić, 2004; Dabroom \& Khalil, 2001) for instance. Numerical schemes can also be applied (Biyık \& Arcak, 2006; Moraal \& Grizzle, 1995), but such observers usually require high computational capacities (Bıyık \& Arcak, 2006) and are only local. An alternative to these approaches is to design a continuoustime observer fed by discrete-time system measures and thus called a continuous-discrete observer. This approach is particularly interesting when the observer dynamics is much faster than the system one (that is the case when high speed processor computers are used). Therefore, the approximation of having a continuous-time observer, although implemented digitally, is acceptable. Moreover, contrary to the discrete-time approach,

\footnotetext{
* The material in this paper was partially presented at the 46th IEEE Conference on Decision and Control, New Orleans. This paper was recommended for publication in revised form by Associate Editor Wei Kang under the direction of Editor André L. Tits.

* Corresponding author. Tel.: +33 0 298348856; fax: +330 298348746

E-mail addresses: tarek.ahmed-ali@greyc.ensicaen.fr (T. Ahmed-Ali) postoyan@lss.supelec.fr (R. Postoyan), lamnabhi@lss.supelec.fr (F. Lamnabhi-Lagarrigue).
}

obtained stability properties are global and asymptotic (in most cases) (Deza, Busvelle, Gauthier, \& Rakotopora, 1992; Hammouri, Kabore, Othman, \& Biston, 2002; Nadri, Hammouri, \& Astorga, 2004) and not semiglobal practical like in the discrete-time formulation (Arcak and Nešić, 2004).

Sampled system measures imply some additional difficulties for the observation compared with the case where outputs are continuously known. Indeed, despite the fact that continuous observation methods have to be adapted to the periodic availability of the output, the system observability also depends on the sampling times. For obvious reasons, if the time interval between two successive measures is too large, the system may lose its observability, as shown in Nadri et al. (2004). Furthermore, considering nonlinear systems with inputs, it is well known that there may exist inputs which make the system unobservable. Hence, the notion of universal input has been introduced to denote the class of inputs which make considered systems observable. This class of inputs, called regularly persistent inputs, has been characterized for some classes of state affine systems in Bornard, Celle, and Couenne (1988), Celle, Gauthier, Kazakos, and Sallet (1989) and Hammouri and Morales (2002).

Existing continuous-discrete time observers are mostly based on extended Kalman filter techniques. In Deza et al. (1992), a class of global exponential observers has been synthesized for multi-input single-output nonlinear systems put into a canonical form. The principle is to divide the observation into two steps: one, called prediction, between sampling times, that consists of 'copying' system dynamics with no correction term and updating the dynamical observation gain, and another one, at the sampling times, where the error between the system and the observer output is used to correct the estimate state trajectory. Deriving 
some conditions on the sampling times through the observer stability analysis, the exponential convergence of the observation error is then ensured. The work of Deza et al. (1992) has been extended to other classes of system. In Nadri and Hammouri (2003), observers for a MIMO class of state affine system where the dynamical matrix depends on the input, have been designed when the inputs are regularly persistent. In Astorga, Othman, Othman, Hammouri, and McKenna (2002), a similar method has been used for a larger class of systems and applied to the observation of emulsion copolymerization process. The observation of a class of systems with output injection has been treated in Nadri et al. (2004). Recently, in Hammouri, Nadri, and Mota (2006), a high gain continuous-discrete observer has been developed but using constant observation gains.

On the other hand, it appears that for a number of physical processes some parameters may not be known, that makes existing results on continuous-discrete time observers non-applicable. In this paper, the observation of the class of systems studied in Nadri and Hammouri (2003) is extended to the adaptive case. Early developments of adaptive observers were made in Kresselmeier (1977) and Lüders and Narendra (1973) for linear systems. Adaptive observation of nonlinear systems has been investigated using different techniques that basically rely on linear adaptive algorithms, through coordinate change or output injection for instance, see Bastin and Gevers (1988), Besançon (2000), Cho and Rajamani (1997), Marino and Tomei (1995) and Zhang (2002). In Zhang (2005), a unified interpretation of the latter references has been proposed that emphasizes their characteristics. In Besançon, León-Morales, and Huerta-Guevara (2006), adaptive observation for state affine systems in continuous-time is discussed and the work (Zhang, 2002) is extended. In this study, the approach developed in Besançon et al. (2006) on extended Kalman filters for a class of MIMO linear time-variant, is adapted to the sampled measures problem at the difference that, here, the estimation law is based on discrete-time adaptive techniques.

After having defined the class of systems considered and recalled the main objective, a class of adaptive continuous-discrete observers is designed. Assuming some persistent excitation conditions and some hypotheses on the system output sampling times to hold, the global exponential stability of the observation error is proved. Finally, a simulation example is performed which illustrates the design procedure.

\section{Nomenclature}

First some mathematical notation is introduced. Let $\mathbb{R} \stackrel{\text { def }}{=}(-\infty$, $\infty), \mathbb{R}_{+} \stackrel{\text { def }}{=}(0, \infty), \mathbb{R}_{0}^{+} \stackrel{\text { def }}{=}[0, \infty)$ and define the Euclidean norm $\|\cdot\|$. For $p, q, n, m \in \mathbb{N}, \mathbb{R}^{p \times q}$ represents the set of real matrices of order $p \times q$ and $\mathbb{I}_{p} \in \mathbb{R}^{p \times p}$ stands for the identity matrix of order $p \times p$. If $\mathcal{X} \subset \mathbb{R}^{p \times q}$ and $\mathcal{Y} \subset \mathbb{R}^{n \times m}, \mathcal{C}(\mathcal{X}, \mathcal{y})$ denotes the space of all continuous functions mapping $X \rightarrow \mathcal{X}$. If $P \in \mathbb{R}^{p \times p}, P>0$ means that $P$ is positive definite. The notation $\|P\|$, for $P \in \mathbb{R}^{p \times q}$, represents the $L_{2}$-norm of $P$. For $A: \mathbb{R} \rightarrow \mathbb{R}^{p \times q}$ and $t \in \mathbb{R}$, the notation $A\left(t^{-}\right)$denotes the left limit of $A$ at instant $t$, if it exists. In all this study, the initial time is called $t_{0} \in \mathbb{R}$.

\section{Problem statement}

The following class of systems is considered, for $t \in\left[t_{k}, t_{k+1}\right)$, $k \geq 0$,

$\dot{x}(t)=A(u) x(t)+b(u)+\phi(u) \theta$,

$y\left(t_{k}\right)=C x\left(t_{k}\right)$,

where $x \in \mathbb{R}^{n}$ is the instantaneous state vector, $u \in \mathscr{D} \subset \mathbb{R}^{m}$ the input vector ( $\mathscr{D}$ is compact), $y \in \mathbb{R}^{p}$ is the output vector, $\theta \in \mathbb{R}^{l}$ is a vector of unknown constant parameters, $A \in \mathcal{C}\left(\mathbb{R}^{m}, \mathbb{R}^{n \times n}\right)$, $b \in \mathcal{C}\left(\mathbb{R}^{m}, \mathbb{R}^{n}\right), C \in \mathbb{R}^{p \times n}, \phi \in \mathcal{C}\left(\mathbb{R}^{m}, \mathbb{R}^{n \times l}\right)$ are known, with $n$, $m, p, l \in \mathbb{N}$ and $x_{0}=x\left(t_{0}\right)$. The notation $\left(t_{k}\right)_{k \geq 0}$ represents a strictly increasing sequence such that $\lim _{k \rightarrow \infty} t_{k}=\infty$ that models the sampling times. The maximum sampling step is denoted $\tau=$ $\max _{k>0}\left(\tau_{k}\right)$, where $\tau_{k}=t_{k+1}-t_{k}$.

Note that the class of systems (1) contains systems of the form, for $k \geq 0$,

$\dot{x}(t)=A(t, u) x(t)+b(t, u)+\phi(t, u) \theta, \quad t \in\left[t_{k}, t_{k+1}\right)$,

$y\left(t_{k}\right)=C x\left(t_{k}\right), \quad t=t_{k+1}$,

since the dependence on $u$ can be considered like a time dependence.

The main objective of this paper is to synthesize a global exponential adaptive observer, as defined in Section 4.3, for system (1).

\section{Observer design and stability study}

\subsection{Observation structure}

The proposed observer can be viewed as an extension to the adaptive case of the structure developed in Nadri and Hammouri (2003). An auxiliary variable, $\lambda$, which plays a key role in the convergence of parameters estimator, is notably introduced as in Besançon et al. (2006) and Zhang (2002).

For $k \geq 0, t \in\left[t_{k}, t_{k+1}\right)$,

$\dot{\hat{x}}(t)=A(u) \hat{x}(t)+b(u)+\phi(u) \hat{\theta}\left(t_{k}\right)$

$\dot{S}(t)=-A(u)^{\mathrm{T}} S(t)-S(t) A(u)-\mu S(t)$,

$\dot{\lambda}(t)=A(u) \lambda(t)+\phi(u)$,

$\dot{\hat{\theta}}(t)=0$,

for $t=t_{k+1}$,

$\begin{aligned} \hat{x}\left(t_{k+1}\right)= & \hat{x}\left(t_{k+1}^{-}\right)+\left(\lambda\left(t_{k+1}\right) \Delta\left(t_{k+1}\right)\right. \\ & \left.+\rho \tau_{k} S^{-1}\left(t_{k+1}\right) C^{\mathrm{T}}\right)\left(y\left(t_{k+1}\right)-C \hat{x}\left(t_{k+1}^{-}\right)\right),\end{aligned}$

$S\left(t_{k+1}\right)=S\left(t_{k+1}^{-}\right)+\tau_{k} C^{\mathrm{T}} C$,

$\lambda\left(t_{k+1}\right)=M\left(\tau_{k}, t_{k+1}\right) \lambda\left(t_{k+1}^{-}\right)$,

$\hat{\theta}\left(t_{k+1}\right)=\hat{\theta}\left(t_{k}\right)+\Delta\left(t_{k+1}\right)\left(y\left(t_{k+1}\right)-C \hat{x}\left(t_{k+1}^{-}\right)\right)$,

with, $\Delta\left(t_{k+1}\right)=\frac{\epsilon}{1+\epsilon\left\|\lambda\left(t_{k+1}^{-}\right)^{\mathrm{T}} C^{\mathrm{T}} \Sigma\left(t_{k+1}^{-}\right) \mathrm{C} \lambda\left(t_{k+1}^{-}\right)\right\|} \lambda\left(t_{k+1}^{-}\right)^{\mathrm{T}} C^{\mathrm{T}} \times \Sigma\left(t_{k+1}^{-}\right)$, and $M\left(\tau_{k}, t_{k+1}\right)=\left(\mathbb{I}_{n}-\rho \tau_{k} S^{-1}\left(t_{k+1}\right) C^{\mathrm{T}} C\right)$. The notation $\hat{x} \in \mathbb{R}^{n}$ and $\hat{\theta} \in \mathbb{R}^{l}$ respectively denote the estimate of the state, $x$, and the estimate of the unknown vector parameter $\theta, S(t) \in \mathbb{R}^{n \times n}$ is the so-called observation gain, $\lambda \in \mathbb{R}^{n}, \Sigma \in \mathcal{C}\left(\mathbb{R}, \mathbb{R}^{p \times p}\right)$ is symmetric positive definite for all $t$, and $\mu, \in \in \mathbb{R}_{+}, \rho \in[1, \infty)$ are some design parameters. Note that the existence of the inverse of matrix $S$ will be ensured in the following. The initial conditions are denoted $\hat{x}_{0} \in \mathbb{R}^{n}, S_{0}>0$ symmetric, $\lambda_{0} \in \mathbb{R}^{n}$ and $\hat{\theta}_{0} \in \mathbb{R}^{l}$.

The observer (3a)-(3d) and (4a)-(4d) is composed of a predictor part (3a)-(3d) and a correction one (4a)-(4d). During $\left[t_{k}, t_{k+1}\right)$, the state estimate $\hat{x}$ has the same dynamics than system (1) but using the estimate of the unknown variable, $\hat{\theta}$, that is kept constant (3d). As commonly done for high gain observers, the matrix $S$ is defined as the solution of the differential equation ( $3 b$ ), except that a term of the form $C^{\mathrm{T}} C$ is usually added in order to ensure $S$ to be positive definite, see for example in Nadri and Hammouri (2003). At each sampling instant, the observer trajectory is corrected taking into 
account the available measure (4a). Thanks to (4b), conditions on the inputs and restriction on $\tau$, it will be shown that matrix $S$ remains positive definite. The dynamics of $\hat{\theta}$ are defined by a least mean squares law (4d), that will guarantee the convergence of the estimate to the true value, under some persistent excitation conditions. The usefulness of variable $\lambda$ will be shown in the stability analysis, as in Besançon et al. (2006) and Zhang (2002).

Remark 1. If the parameter vector $\theta$ is equal to zero, the system (3a)-(3d) and (4a)-(4d) has the same form as in Nadri and Hammouri (2003).

The dynamical equation satisfied by the state observation error, $e=x-\hat{x}$, is, in view of (1), (3a)-(3d) and (4a)-(4d), for $k \geq 0$ :

$$
\begin{gathered}
\dot{e}(t)=A(u) e(t)+\phi(u) \tilde{\theta}\left(t_{k}\right), \quad t \in\left[t_{k}, t_{k+1}\right), \\
e\left(t_{k+1}\right)=\left(M\left(\tau_{k}, t_{k+1}\right)-\lambda\left(t_{k+1}\right) \Delta\left(t_{k+1} C\right)\right) e\left(t_{k+1}^{-}\right), \\
t=t_{k+1} .
\end{gathered}
$$

The following persistent excitation condition is assumed to hold for system (1) in order to guarantee the identifiability of vector $\theta$ (Besançon et al., 2006; Zhang, 2002).

Hypothesis 2. Let $\lambda(\cdot) \in \mathbb{R}^{n \times s}$ be the matrix solution of the impulsive ordinary differential equations (3c), (3d) and (4a)-(4c). The matrix $\lambda(\cdot)$ is persistently excited so that there exist $k_{1} \in \mathbb{N}, \delta>0$ and $\Sigma(\cdot) \in \mathcal{C}\left(\left[t_{0}, \infty\right), \mathbb{R}^{p \times p}\right)$ a time-dependent bounded symmetric positive definite matrix, such that, for all $k \geq 0$,

$\sum_{j=k}^{k+k_{1}} \lambda^{\mathrm{T}}\left(t_{j}^{-}\right) C^{\mathrm{T}} \Sigma\left(t_{j}\right) C \lambda\left(t_{j}^{-}\right) \geq \delta \mathbb{I}_{n}$.

Before stating the main theorem, some preliminary definitions and results are required.

Definition 3. Consider the system, for $t \in\left[t_{0}, \infty\right)$ :

$\dot{x}(t)=A(u) x(t)$

$y(t)=C x(t)$

and the transition matrix $\Psi_{u}\left(\cdot, t_{0}\right)$ associated to system (7), such that $\Psi_{u}\left(t_{0}, t_{0}\right)=\mathbb{I}_{n}$, the bounded input $u$ is said to be regularly persistent if there exist $t_{1}, t_{2} \in\left[t_{0}, \infty\right), \alpha \in \mathbb{R}_{+}$, such that, for all $t \geq t_{2}$,

$\int_{t}^{t+t_{1}} \Psi_{u}^{\mathrm{T}}\left(s, t_{0}\right) C^{\mathrm{T}} C \Psi_{u}\left(s, t_{0}\right) \mathrm{d} s \geq \alpha \mathbb{I}_{n}$.

As mentioned in the Introduction, regularly persistent inputs are the class of inputs that guarantee the system to be observable. For more details, see Bornard et al. (1988), Celle et al. (1989) and Hammouri and Morales (2002).

Remark 4. In practice, condition (8) can be checked off-line, when $u(t)$ is known for all $t \in\left[t_{0}, \infty\right)$, using numerical tools for approximating the integral term. Thus, coefficients $t_{1}$ and $\alpha$ are obtained off-line in order to derive a sampling period that satisfies the bound given in (9).

\subsection{Technical results}

The following proposition guarantees that matrix $S$ is positive definite for any $t \in\left[t_{0}, \infty\right)$, under conditions on the types of inputs applied to the system and $\tau$.
Proposition 5. Let $u$ be a regularly persistent input for system (1), for all $\mu \geq \xi$ where $\xi=2 \sup _{t \geq t_{0}}\|A(u(t))\|$, if $\tau \leq \bar{\tau}$, where $\bar{\tau}$ is the unique positive term such that $\bar{\tau}=\frac{\alpha \mathrm{e}^{-\xi\left(t_{1}+\bar{\tau}\right)}}{2 \xi\left\|C^{\mathrm{T} C}\right\|\left(t_{1}+\bar{\tau}\right)}$, then, for all $S\left(t_{0}\right)$ symmetric positive definite, there exist constants $\beta_{1}, \beta_{2} \in \mathbb{R}_{+}$such that, for $t \in\left[t_{0}, \infty\right)$,

$\beta_{1} \mathbb{I}_{n} \leq S(t) \leq \beta_{2} \mathbb{I}_{n}$. (2003).

The proof of Proposition 5 can be found in Nadri and Hammouri

Remark 6. (a) Since considered inputs belong to a compact set, $D$, and because $A$ is continuous, $\xi$ is well defined.

(b) There always exists such a $\bar{\tau}$; indeed writing condition $\bar{\tau}=$ $\frac{\alpha \mathrm{e}^{-\bar{\xi}\left(t_{1}+\bar{\tau}\right)}}{2 \xi\left\|C^{\mathrm{T}} C\right\|\left(t_{1}+\bar{\tau}\right)}$ as $2 \xi\left\|C^{\mathrm{T}} C\right\|\left(t_{1}+\bar{\tau}\right) \bar{\tau}=\alpha \mathrm{e}^{-\xi\left(t_{1}+\bar{\tau}\right)}$, loosely speaking it is clear that, for positive values of $\bar{\tau}$, the polynomial term on the left-hand side will 'cross' the exponential on the righthand side at a unique point.

(c) Considering regularly persistent inputs, the positive definition of the solution $S$ of Eqs. (3b)-(3d), (4a) and (4b) can only be ensured under condition on the sampling steps, otherwise the solution of Eq. (3b) may become non-positive definite (Nadri \& Hammouri, 2003).

(d) Notice that $\beta_{1}, \beta_{2}$ implicitly depend on the maximum allowable sampling interval $\bar{\tau}$.

Lemma 7. Suppose the following system to be globally exponentially stable,

$\zeta\left(t_{k+1}\right)=F\left(t_{k}\right) \zeta\left(t_{k}\right)$

and let $u$ be a bounded and integrable function of time that vanishes exponentially fast, then, system

$z\left(t_{k+1}\right)=F\left(t_{k}\right) z\left(t_{k}\right)+u\left(t_{k}\right), \quad k \geq 0$,

converges exponentially to 0 .

Remark 8. This lemma is a straight extension of Lemma III.1, in Marino and Tomei (1995), for discrete-time systems.

\subsection{Stability analysis}

System (3a)-(3d) and (4a)-(4d) is said to be a global exponential adaptive observer for system (1) if, along solutions to (1), (3a)-(3d) and $(4 a)-(4 d)$ :

(i) for all $\left(x_{0}, \hat{x}_{0}, \lambda_{0}, \hat{\theta}_{0}\right) \in \mathbb{R}^{n} \times \mathbb{R}^{n} \times \mathbb{R}^{n} \times \mathbb{R}^{l}$ and any $S_{0}>0$ symmetric, there exist $a_{1}, a_{2}, b_{1}, b_{2} \in \mathbb{R}_{+}$, such that for all $t \geq$ $t_{0},\|e(t)\| \leq a_{1} \mathrm{e}^{-b_{1}\left(t-t_{0}\right)}\left\|e_{0}\right\|$ and $\|\tilde{\theta}(t)\| \leq a_{2} \mathrm{e}^{-b_{2}\left(t-t_{0}\right)}\left\|\tilde{\theta}_{0}\right\|$.

(ii) there exist $\bar{\lambda}, \bar{S} \in \mathbb{R}_{+}$, such that, for all $t \geq t_{0},\|\lambda(t)\| \leq \bar{\lambda}$, $\|S(t)\| \leq \bar{S}$.

Condition (i) ensure the exponential convergence of the state and variable estimates to the corresponding true value, whereas (ii) ensure that the other observer variables do not explode in (in)finite time.

Theorem 9. Assuming Hypothesis 2 to hold, the input to be regularly persistent, and choosing $\mu \geq \xi$ if,

$\tau \leq \min \left\{\frac{(2 \rho-1) \beta_{1}}{\rho^{2}\|C\|^{2}}, \bar{\tau}\right\}$,

then system (3a)-(3d) and (4a)-(4d) is a global exponential adaptive observer for system (1). 
Proof. As in Besançon et al. (2006) and Zhang (2002), the variable $\eta$ is introduced as $\eta=e-\lambda \tilde{\theta}$, where $\tilde{\theta}=\theta-\hat{\theta}$. First, the global exponential convergence of $\eta$ to zero, along the solutions to dynamical equations to (1), (3a)-(3d) and (4a)-(4d), is proved. After having noticed that variables $\lambda$ and $S$ remain bounded, invoking Lemma 7, the exponential convergence to the origin of errors $e$ and $\tilde{\theta}$ is deduced.

The candidate Lyapunov function is defined as, for $t \geq t_{0}$ :

$V(t)=\eta(t)^{\mathrm{T}} S(t) \eta(t)$.

Note that, according to Proposition 5 and since (9) is satisfied, the symmetric matrix $S$ is positive definite and of bounded norm. Let $k \geq 0$.

1. Let $t \in\left[t_{k}, t_{k+1}\right)$. The variable $\eta$ satisfies the following dynamical equation, in view of (5a) and because, here, $\dot{\tilde{\theta}}=0$ (3d):

$$
\begin{aligned}
\dot{\eta}(t) & =A(u) e(t)+\phi(u) \tilde{\theta}\left(t_{k}\right)-\dot{\lambda}(t) \tilde{\theta}\left(t_{k}\right) \\
& =A(u) \eta(t)+(A(u) \lambda(t)+\phi(u)-\dot{\lambda}(t)) \tilde{\theta}\left(t_{k}\right) .
\end{aligned}
$$

Thus, according to $(3 \mathrm{c})$,

$\dot{\eta}(t)=A(u) \eta(t)$.

Consequently, differentiating the Lyapunov function along the solutions of $(10)$,

$\dot{V}(t)=\eta(t)^{\mathrm{T}}\left(S(t) A(u)+A(u)^{\mathrm{T}} S(t)+\dot{S}(t)\right) \eta(t)$.

From (3b),

$\dot{V}(t)=-\mu V(t)$.

Integrating (11) over $\left[t_{k}, t\right]$,

$V(t)=\mathrm{e}^{-\mu\left(t-t_{k}\right)} V\left(t_{k}\right)$,

and so,

$V\left(t_{k+1}^{-}\right)=\mathrm{e}^{-\mu \tau_{k}} V\left(t_{k}\right)$.

2. Consider now $t=t_{k+1}$, it can be shown that, in view of (5b),

$$
\begin{aligned}
\eta\left(t_{k+1}\right)= & \left(M\left(\tau_{k}, t_{k+1}\right)-\lambda\left(t_{k+1}\right) \Delta\left(t_{k+1}\right) C\right) e\left(t_{k+1}^{-}\right) \\
& -\lambda\left(t_{k+1}\right) \tilde{\theta}\left(t_{k+1}\right) \\
= & M\left(\tau_{k}, t_{k+1}\right)\left(\eta\left(t_{k+1}^{-}\right)+\lambda\left(t_{k+1}^{-}\right) \tilde{\theta}\left(t_{k+1}^{-}\right)\right) \\
& -\lambda\left(t_{k+1}\right)\left(\Delta\left(t_{k+1}\right) C e\left(t_{k+1}^{-}\right)+\tilde{\theta}\left(t_{k+1}\right)\right) .
\end{aligned}
$$

Remarking that $\tilde{\theta}\left(t_{k+1}^{-}\right)=\tilde{\theta}\left(t_{k}\right)$ in view of $(3 \mathrm{~d})$, and using (4b)-(4d),

$\eta\left(t_{k+1}\right)=M\left(\tau_{k}, t_{k+1}\right) \eta\left(t_{k+1}^{-}\right)$.

According to (4d),

$$
\begin{aligned}
V\left(t_{k+1}\right)= & \eta\left(t_{k+1}\right)^{\mathrm{T}} S\left(t_{k+1}\right) \eta\left(t_{k+1}\right) \\
= & \eta\left(t_{k+1}^{-}\right)^{\mathrm{T}} M\left(\tau_{k}, t_{k+1}\right)^{\mathrm{T}}\left(S\left(t_{k+1}^{-}\right)+\tau_{k} C^{\mathrm{T}} C\right) \\
& \times M\left(\tau_{k}, t_{k+1}\right) \eta\left(t_{k+1}^{-}\right) \\
= & V\left(t_{k+1}^{-}\right)-(2 \rho-1) \tau_{k}\left\|C \eta\left(t_{k+1}^{-}\right)\right\|^{2} \\
& +\rho^{2} \tau_{k}^{2} \eta\left(t_{k+1}^{-}\right) C^{\mathrm{T}} C S^{-1}\left(t_{k+1}\right) C^{\mathrm{T}} C \eta\left(t_{k+1}^{-}\right) .
\end{aligned}
$$

Invoking Proposition 5, the following inequality holds:

$$
\begin{aligned}
V\left(t_{k+1}\right) \leq & V\left(t_{k+1}^{-}\right)-\tau_{k}\left((2 \rho-1)-\frac{\rho^{2} \tau_{k}\|C\|^{2}}{\beta_{1}}\right) \\
& \times\left\|C \eta\left(t_{k+1}^{-}\right)\right\|^{2} .
\end{aligned}
$$

Using (13) and because (9) holds,

$V\left(t_{k+1}\right) \leq \mathrm{e}^{-\mu \tau_{k}} V\left(t_{k}\right)$.
Consequently, according to (12) and (16), for all $t \geq t_{0}$,

$V(t) \leq \mathrm{e}^{-\mu\left(t-t_{0}\right)} V\left(t_{0}\right)$,

$\|\eta(t)\| \leq \sqrt{\frac{\beta_{2}}{\beta_{1}}} \mathrm{e}^{-\frac{\mu}{2}\left(t-t_{0}\right)}\left\|\eta\left(t_{0}\right)\right\|$,

the exponential stability of $\eta=0$ for system (10)-(14) is proved. Using similar arguments than for variable $\eta$, in view of (3c) and (4c) and since function $\phi$ is continuous and $u$ takes values in a compact set, it can be easily shown that there exists $\bar{\lambda} \in \mathbb{R}_{+}$such that $\|\lambda(t)\| \leq \bar{\lambda}$, for $t \geq t_{0}$. On the other hand, according to (4d),

$\tilde{\theta}\left(t_{k+1}\right)=\left(\mathbb{I}_{n}-\Delta\left(t_{k+1}\right) C \lambda\left(t_{k+1}^{-}\right)\right) \tilde{\theta}\left(t_{k}\right)-\Delta\left(t_{k+1}\right) C \eta\left(t_{k+1}^{-}\right)$.

Attention is focused on the homogenous part of (17), which is typical of the normalized least mean squares algorithm:

$\tilde{\theta}\left(t_{k+1}\right)=\left(\mathbb{I}_{n}-\Delta\left(t_{k+1}\right) C \lambda\left(t_{k+1}^{-}\right)\right) \tilde{\theta}\left(t_{k}\right)$.

Using Theorem 2.8 in Anderson et al. (1986), since Hypothesis 2 holds, $\tilde{\theta}=0$ is globally exponentially stable for system (18). The non-homogenous part of Eq. (17) vanishes exponentially fast, because of the exponential convergence of $\eta$ to 0 and the boundedness of $\Delta$. Therefore, applying Lemma 7, $\tilde{\theta}=0$ is globally exponentially stable for system (17). Writing the observation error as $e=$ $\eta+\lambda \tilde{\theta}$, the exponential convergence of $e$ to 0 , along the solutions of (5a) and (5b), can be deduced from the facts that $\tilde{\theta}$ and $\eta$ converge with an exponential rate to zero and that $\lambda$ is bounded.

Remark 10. Throughout the paper, no stability assumption is supposed to hold for $A$. In view of Theorem 9, even unstable matrices can be considered.

Remark 11. The sampling interval must satisfy (9). In practice, after having numerically found admissible $t_{1}$ and $\alpha$, the upper bound in (9) can be determined using parameters values.

\subsection{Discussion on parameters choice}

The selection of the observer parameters $\mu, \rho, \epsilon$ illustrates the logical compromise between convergence speed and bound on $\tau$.

As it can be seen through (3b), parameter $\mu$ (taken bigger that $\xi$ in view of Theorem 9) acts on the observation gain in such a way that taking big values will increase the convergence speed of the observer at the price of a smaller admissible $\tau$. Indeed, this will make the solution of ( $3 b$ ) go faster to non-positive definite matrices and then decrease the value of $\beta_{1}$ (see Remark 6(d)). Concerning $\rho$, a quick analysis could conclude that $\rho=1$ is the optimal value to be chosen in view of (9), but a bigger value will help $\eta$ to converge faster to 0 , again, under more severe conditions on $\tau$ (see (14) and the definition of $M$ ). On the other hand, parameter $\varepsilon$ allows one to modulate the convergence of the parameter estimate.

\section{Illustrative example}

For illustrative purposes, consider a second-order system modeled by, for $k \geq 0$ and $t \in\left[t_{k}, t_{k+1}\right)$,

$\dot{x}_{1}=\sin (u) x_{2}$

$\dot{x}_{2}=-0.1 \sin (u) x_{1}-0.2 x_{2}+0.1 \cos (u) \theta$,

$y=x_{1}$,

where $\theta$ is unknown and $u(t)=2-(\cos (0.1 t))^{2}$. The sampling step is chosen constant at $\tau=0.11$. An observer of the form (3a)-(3d) and (4a)-(4d) is designed for system (19). The design parameters have been fixed at $\rho=1, \epsilon=1, \mu=2.1$ and $\Sigma=1$. Using numerical tools, the following values have been found $\alpha=0.5$, 

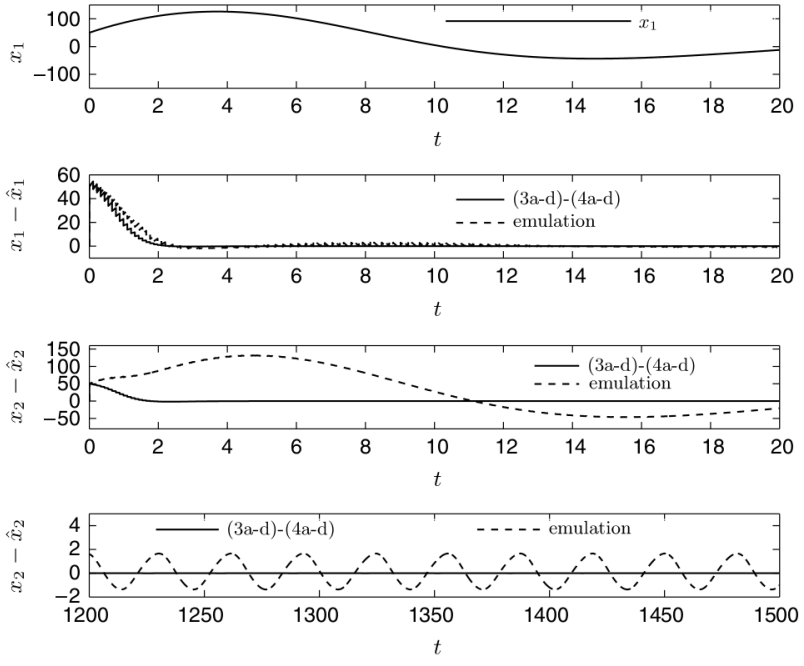

Fig. 1. State $x_{1}$ and convergence of the state observation errors.
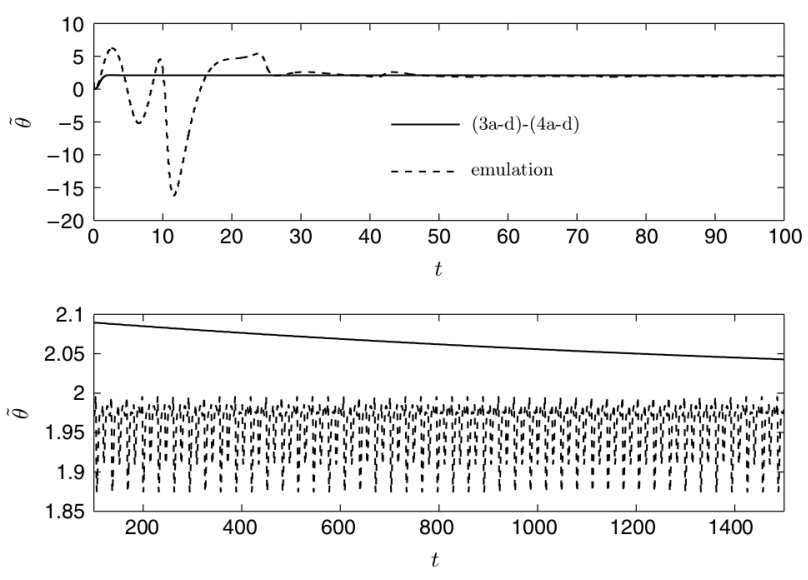

Fig. 2. Convergence of the parameter estimate.

$t_{1}=0.01, \xi=2.0396, \beta_{1} \geq 0.1125$, consequently, condition (9) is satisfied: $\tau \leq \min \{0.1125,0.21\}$. Therefore, since the input is a persistent input for system (19), Theorem 9 applies. The initial conditions are $t_{0}=0, x_{1}(0)=x_{2}(0)=50, \hat{x}_{1}(0)=\hat{x}_{2}(0)=\hat{\theta}(0)=0$ and $\theta=2$. For comparative purpose, the Euler discretization of observer (11-15) in Besançon et al. (2006) has been simulated. Fig. 1 shows that, contrary to the continuous-time observer, the state $\hat{x}_{2}$ given by the emulation does not converge asymptotically to $x_{2}$ but oscillates. As mentioned in the Introduction, this fact is not surprising since only semiglobal practical stability properties can be established when using the emulation of a continuous-time observer (Arcak and Nešić, 2004). Moreover, the behavior of emulation states present a big overshoot by opposite to the results obtained by the observer (3a)-(3d) and (4a)-(4d). Obtained parameter estimates converge slowly asymptotically to the true value for the continuous-discrete observer, and again, practically and with large overshoots for the emulation (Fig. 2). Although not presented here, simulation results show that the continuous-time observer still works efficiently for larger values of $\tau$ like $\tau=3$ whereas, for such a value, the emulation states explode.

\section{Conclusions}

The adaptive observation of a class of continuous MIMO systems with sampled measurements has been realized. Assuming that the input acts on the system to satisfy some persistent excitation conditions and the sampling steps to respect given bounds, a class of global exponential observers has been developed.

\section{References}

Anderson, B. D. O., Bitmead, R. R., Johnson, C. R., Kokotović, P. V., Kosut, R. L., Mareels, I. M. Y., et al. (1986). Signal processing, optimization and control, Stability of adaptive systems: Passivity and averaging analysis. Cambridge, USA: MIT Press.

Arcak, M., \& Nešić, D. (2004). A framework for nonlinear sampled-data observer design via approximate discrete-time models and emulation. Automatica, 40 1931-1938.

Astorga, C.-M., Othman, N., Othman, S., Hammouri, H., \& McKenna, T.-F. (2002). Nonlinear continuous-discrete observers: Applications to emulsion polymerization reactors. Control Engineering Practice, 10, 3-13.

Bastin, G., \& Gevers, M. (1988). Stable adaptive observers for nonlinear time varying systems. IEEE Transactions on Automatic Control, 33(7), 650-658.

Besançon, G. (2000). Remarks on nonlinear adaptive observer design. Systems \& Control Letters, 41(7), 271-280.

Besançon, G., León-Morales, J. D., \& Huerta-Guevara, O. (2006). On adaptive observers for state affine systems. International Journal of Control, 79(6), 581-591.

Biyık, E., \& Arcak, M. (2006). A hybrid redesign of newton observers in the absence of an exact discrete-time model. Systems $\mathcal{E}$ Control Letters, 55, 429-436.

Bornard, G., Celle, F., \& Couenne, N. (1988). Regularly persistent observers for bilinear systems. In Proc. of the 29th international conference on nonlinear systems, New trends in nonlinear systems theory (pp. 130-140). Vol. 122.

Celle, F., Gauthier, J. P., Kazakos, D., \& Sallet, G. (1989). Synthesis of nonlinear observers: A harmonic-analysis approach. Mathematical Systems Theory, 22, 291-322.

Cho, Y. M., \& Rajamani, R. (1997). A systematic approach to adaptive observer synthesis for nonlinear systems. IEEE Transactions on Automatic Control, 42, 534-537.

Dabroom, A. M., \& Khalil, H. K. (2001). Output feedback sampled-data control of nonlinear systems using high-gain observers. IEEE Transactions on Automatic Control, 46(11), 1712-1725.

Deza, F., Busvelle, E., Gauthier, J. P., \& Rakotopora, D. (1992). High gain estimation for nonlinear systems. Systems \& Control Letters, 18, 295-299.

Hammouri, H., Kabore, P., Othman, S., \& Biston, J. (2002). Failure diagnosis and nonlinear observer. Application to a hydraulic process. Journal of The Franklin Institute, 339, 455-478.

Hammouri, H., \& Morales, J. D. (2002). Topological properties of observer's inputs. In Progress in syst. and control theory. Boston, USA: Birkhauser.

Hammouri, H., Nadri, M., \& Mota, R. (2006). Constant gain observer for continuous-discrete time uniformly observable systems. In Proc. of the 45th IEEE conf. on dec. E' control (pp. 6240-6244).

Kresselmeier, G. (1977). Adaptive observers with exponential rate of convergence. IEEE Transactions on Automatic Control, AC-22, 2-8.

Lüders, G., \& Narendra, K. (1973). An adaptive observer and identifier for a linear system. IEEE Transactions on Automatic Control, AC-18, 496-499.

Marino, R., \& Tomei, P. (1995). Adaptive observers with arbitrary exponential rate of convergence for nonlinear systems. IEEE Transactions on Automatic Control, 40 , 1300-1304.

Marino, R., \& Tomei, P. (1995). Information and system sciences, Nonlinear control design. London, UK: Prentice Hall.

Moraal, P. E., \& Grizzle, J. W. (1995). Observer design for nonlinear systems with discrete-time measurements. IEEE Transactions on Automatic Control, 40(3), 395-404.

Nadri, M., \& Hammouri, H. (2003). Design of a continuous-discrete observer for state affine systems. Applied Mathematics Letters, 16, 967-974.

Nadri, M., Hammouri, H., \& Astorga, C.-M. (2004). Observer design for continuous-discrete time state affine systems up to ouput injection. European Journal of Control, 10, 252-263.

Zhang, Q. (2002). Adaptive observer for multi-input-multi-ouput (mimo) linear time-varying systems. IEEE Transactions on Automatic Control, 47(3), 525-529.

Zhang, Q. (2005). Revisiting different adaptive observers through a unified formulation. In Proc. of the 45th IEEE conf. on dec. E' control and eur. conf. on control (pp. 3067-3072). 